\title{
Comportamento de cultivares de soja, em diferentes épocas de semeaduras, visando a produção de biocombustivel ${ }^{1}$
}

\author{
Behaviour of soybean cultivars, at different sowing seasons, aiming to the production \\ of biofuel
}

\begin{abstract}
Vitor da Silva Barbosa ${ }^{2}$, Joenes Mucci Peluzio ${ }^{3 *}$, Flávio Sérgio Afférri ${ }^{3}$ Guilherme Benko de Siqueira ${ }^{3}$
\end{abstract}
\begin{abstract}
Resumo - A época de semeadura é definida por um conjunto de fatores ambientais que reagem entre si e interagem com a planta, promovendo variações na produção e afetando outras características agronômicas. Assim, o trabalho teve como objetivo avaliar o comportamento de 21 cultivares comerciais de soja, em diferentes épocas de semeadura, visando a produção de biocombustível. Foram conduzidos cinco ensaios no ano agrícola 2008/09, sendo dois na área experimental da Universidade Federal do Tocantins UFT, em Palmas-TO e três na Área Experimental da UFT, em Gurupi-TO. Os plantios foram efetuados em 03/12/2008, 18/12/2008 e 05/01/2009, em Gurupi, e 30/11/2009 e 16/12/2009, em Palmas. O delineamento experimental foi de blocos ao acaso com 21 tratamentos e três repetições. Os tratamentos foram constituídos pelos cultivares P98Y70, M-SOY 8766RR, M-SOY 9144RR, BR/Emgopa 314, P98R91, P98Y51, M-SOY 9988RR, P99R01, M-SOY 8867RR, M-SOY 9056RR, M-SOY 8527RR, M-SOY 8360RR, FT ESPERANÇA, FTS 4188, CM 07, CM 017, CM 136, CM 149, CM 102, NIDERA A 7002 e M-SOY 9350. Foram avaliadas as características: rendimento de grãos, teor de óleo e rendimento de óleo. Foram detectadas diferenças significativas entre cultivares, épocas e cultivares x épocas para todas as características. A primeira época de plantio em Palmas (30/11) e Gurupi $(03 / 12)$ foram as mais propícias para o desenvolvimento dos cultivares. Houve associação positiva e significativa entre rendimento de óleo e produção de grãos $(\mathrm{R}=0,97)$. Temperaturas mais altas e menores médias de precipitações pluviométricas durante a fase de enchimento de grãos favoreceram o acúmulo de óleo nas sementes. Os cultivares que mais se destacaram para teor de óleo e rendimento de óleo foram NIDERA A 7002, MSOY 9144RR, BR/EMGOPA 314, P98Y51 e FTS 4188.
\end{abstract}

Palavras-chaves - Soja. Agricultura e energia. Biocombustíveis. Semeadura.

\begin{abstract}
The sowing season is defined by a set of environmental factors that react with each other and interact with the plant, thus affecting variations in production and other agronomic traits. This work was carried out to evaluate the behavior of 21 cultivars of commercial soybean, during different times, looking forward to the production of biofuel. Were conducted five essays in the 2008/09 agricultural year, being two of them in Palmas-TO and three in Gurupi-TO. The sowing in Gurupi-TO was in 03/12/08, 18/12/2008 and 05/01/2009, and in Palmas in 30/11/2009 and 16/12/2009. The experimental design was a randomized blocks with 21 treatments and three repetitions. The treatments were constituted on the cultivars P98Y70, M-SOY 8766RR, M-SOY 9144RR, BR/Emgopa 314, P98R91, P98Y51, M-SOY 9988RR, P99R01, M-SOY 8867RR, M-SOY 9056RR, M-SOY 8527RR, M-SOY 8360RR, FT ESPERANÇA, FTS 4188, CM 07, CM 017, CM 136, CM 149, CM 102, NIDERA A 7002 and M-SOY 9350. The following characteristics, were evoluated production of grains, oil content of the grain and oil yield. Significant differences were detected among cultivars, sowing season and cultivate $\mathrm{x}$ sowing season for all characteristics. The first planting time in Palmas (30/11) and Gurupi (03/12), were the most favorable to the development of cultivars. There was positive and significant association between oil yield and production of grains $(R=0.97)$. Higher temperatures and rainfall precipitation averages, during the phase of stuffing of grains, favored the oil accumulation in the grains. NIDERA A 7002, MSOY 9144RR, BR/Emgopa 314, P98Y51 and FTS 4188 were the best cultivars for oil content of the grain and oil yield.
\end{abstract}

Key word - Soybean. Agriculture and energy. Sowing. Biofuels.

\footnotetext{
* Autor para correspondência

${ }^{1}$ Recebido para publicação em 12/05/2010; aprovado em 13/04/2011

Parte da Dissertação de Mestrado do primeiro autor apresentada na Fundação Universidade Federal do Tocantins, Campus Universitário de Palmas ${ }^{2}$ Programa de Pós-Graduação em Agroenergia/UFT, Campus Universitário de Palmas, Palmas-TO, Brasil, 77.402-970, barbosavs@ hotmail.com ${ }^{3}$ Universidade Federal do Tocantins, Campus Universitário de Palmas, Caixa Postal 66, Palmas-TO, Brasil, 77.402-970, joenesp@uft.edu.br, flavio@ mail.uft.edu.br, guibenko@uft.edu.br
} 


\section{Introdução}

A utilização de biodiesel como combustível tem sido promissor no mundo inteiro, por efeito de um mercado crescente, devido: a) a sua enorme contribuição ao meio ambiente, com a redução qualitativa e quantitativa dos níveis de poluição ambiental, principalmente, nos grandes centros urbanos; b) como fonte estratégica de energia renovável em substituição ao óleo diesel e outros derivados do petróleo (CLEMENTE; CAHOON, 2009; KINNEY; CLEMENTE, 2005; SILVA; FREITAS, 2008).

Os programas nacionais de biocombustíveis vêm estimulando a oferta de óleos vegetais no mercado. A soja, por apresentar ampla adaptação climática no Brasil (Norte-Sul), amplo domínio tecnológico (óleo e proteína - multiuso), logística favorável e resistência a flutuações de preços do mercado, refletindo em alta produção e oferta, responde por $90 \%$ da matéria prima para produção de biodiesel no Brasil (FERRARI et al., 2005; PÍPOLO, 2002; RANGEL et al., 2004).

No Estado do Tocantins, a soja é a terceira cultura de maior importância econômica, sendo cultivada no período de entressafra (maio-junho), em condições de várzea irrigada, sob regime de sub-irrigação (elevação do lençol freático), principalmente, em Formoso do Araguaia, e no período de safra (novembro-dezembro), em condições de terras altas (PELUZIO et al., 2006). Entretanto, apesar da grande importância da cultura no Estado, há escassez de pesquisas relacionadas à influência da época de semeadura em características agronômicas nas cultivares, principalmente, em relação às características teor e rendimento de óleo.

Os programas de melhoramento de soja têm se concentrado no desenvolvimento de variedades mais produtivas. Entretanto, mais recentemente, ha também uma preocupação com características de qualidade, tais como conteúdo e composição do óleo e da proteína de reserva. Para isto, o melhorista tem recorrido ao germoplasma, tanto o adaptado como o silvestre, à busca de variabilidade genética. Esses novos materiais e as linhagens derivadas precisam ser caracterizados sob o ponto de vista fisiológico, o que demanda um longo período. Assim, com o intuito de se ganhar tempo, trabalhos de pesquisa tem sido realizados visando testar a capacidade de produção de óleo dos cultivares comerciais destinadas à indústria alimentícia (óleo e proteína).

Como o desempenho relativo das características agronômicas das cultivares (produção de grãos, teor e rendimento de óleo, dentre outras) pode variar de um ambiente de cultivo para outro, faz-se necessário um estudo multi-ambiental, ou seja, que diferentes cultivares sejam testadas em diferentes locais, anos, épocas, tecnologias, etc (CRUZ; REGAZZI, 2006; MAEHLER et al., 2003; MINUZZI et al., 2009; PEREIRA et al., 2008; SILVA; DUARTE, 2006).

Os teores de óleo e proteína dos grãos de soja são governados geneticamente, porém fortemente influenciados pelo ambiente, principalmente, durante o período de enchimento dos grãos (ALBRECHT et al., 2008; MINUZZI et al., 2009; PÍPOLO, 2002; RANGEL et al., 2004; RAO et al., 1993). Segundo Albrecht et al.(2008), Minuzzi et al.(2009), Pípolo (2002) e Rangel et al.(2004), variações na temperatura que ocorrem aos 20-40 dias antes da maturação dos grãos exercem maior influência sobre o acúmulo de óleo do que às ocorridas em outros períodos.

Diante disso, o presente trabalho objetivou avaliar o comportamento de 21 cultivares comerciais de soja, em diferentes épocas de semeadura, visando a produção de biocombustivel, na Região Centro-Sul do Estado do Tocantins - safra 2008/2009.

\section{Material e métodos}

Foram realizados cinco ensaios de competição de cultivares no ano agrícola 2008/09, sendo dois na área experimental da Universidade Federal do Tocantins em

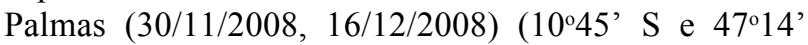
$\mathrm{W}, 220 \mathrm{~m})$ e três na área experimental da Universidade Federal do Tocantins em Gurupi (03/12/2008, 18/12/2008, $05 / 01 / 2009)\left(11^{\circ} 43^{\prime} \mathrm{S}\right.$, e $\left.49^{\circ} 04^{\prime} \mathrm{W}, 280 \mathrm{~m}\right)$, em solo do tipo Latossolo vermelho amarelo. Cada ensaio foi resultante da combinação época/local.

O delineamento experimental utilizado foi de blocos casualizados, com 21 tratamentos e três repetições. Os tratamentos foram constituídos pelas cultivares P98Y70, MSOY 8766RR, M-SOY 9144RR, BR/Emgopa 314, P98R91, P98Y51, MSOY 9988RR, P99R01, M-SOY 8867RR, MSOY 9056RR, M-SOY 8527RR, M-SOY 8360RR, FT Esperança, FTS 4188, CM 015, CM 017, CM 136, CM 149, CM 102, NIDERA A 7002, M-SOY 9350, tradicionalmente cultivadas no período visando a produção de grãos.

A parcela experimental foi composta por quatro fileiras de 5,0 $\mathrm{m}$ de comprimento, espaçadas por $0,45 \mathrm{~m}$. Foram colhidas as duas fileiras centrais, sendo desprezados $0,50 \mathrm{~m}$ da extremidade de cada fileira.

Foram realizadas as operações de aração, gradagem e sulcamento. A adubação de plantio foi realizada conforme as exigências da cultura, após análise prévia do solo.

No momento do plantio, foi realizado o tratamento das sementes com fungicidas, seguido de inoculação das sementes com estirpes de Bradyrhizobium japonicum. 
A densidade de semeadura foi realizada com intuito de se obter 14 plantas por metro linear. O controle de pragas, doenças e plantas daninhas foi realizado à medida que se fizeram necessários.

As plantas, de cada parcela experimental, foram colhidas uma semana após terem apresentado $95 \%$ das vagens maduras, ou seja, no estádio $R_{8}$ da escala de Fehr et al. (1971). Após a colheita, as plantas foram trilhadas e as sementes pesadas, depois de secas (12\% de umidade) e limpas, para a determinação dos rendimentos de grãos.

Com base na área útil da parcela, foi determinado o rendimento de grãos (peso em $\mathrm{kg} \mathrm{ha}^{1}$, após a correção da umidade para $12 \%$ ). Posteriormente, foi determinado o teor de óleo dos grãos (\%) e o rendimento de óleo $\left(\mathrm{kg} \mathrm{ha}^{-1}-\%\right.$ óleo x rendimento de grãos), no laboratório do Curso de Engenharia de Alimentos da Universidade Federal do Tocantins - Campus de Palmas, através do Método de Soxhlet (Instituto Adolfo Lutz, 1985), por ser prático e exequível. Foram obtidas três amostras por cultivar, em cada um dos ensaios, cada uma pesando de 2 a 5 gramas.

Com os dados das características, foi realizada análise de variância individual e, posteriormente, a análise conjunta dos ensaios em que o menor quadrado médio residual não diferiu em mais de sete vezes do maior (CRUZ; REGAZZI., 2006), utilizando-se o programa estatístico SISVAR (UFLA). As médias dos tratamentos foram comparadas pelo teste de Scott Knott, ao nível de $5 \%$ de significância.

\section{Resultados e discussão}

A análise de variância conjunta (TAB. 1) apresentou efeito significativo de cultivar, época e da interação cultivar $\mathrm{x}$ época para todas as características, esta última indicando que os efeitos isolados dos fatores cultivares e épocas não explicam toda a variação encontrada. Assim, foram realizados os desdobramentos. Os coeficientes de variação foram baixos, indicando a boa precisão do experimento.

\section{Rendimento de Grãos}

Para a característica rendimento de grãos (TAB. 2), o plantio realizado em Palmas (30/11) alcançou a maior média geral (4222 $\left.\mathrm{kg} \mathrm{ha}^{-1}\right)$, diferindo, significativamente, do plantio efetuado em Gurupi (03/12) (2936 kg ha-1). No plantio realizado em 30/11, a cultivar P98R91 apresentou a maior média de rendimento de grãos $\left(5050 \mathrm{~kg} \mathrm{ha}^{-1}\right)$, seguida de BR/Emgopa 314 (4983 $\mathrm{kg} \mathrm{ha}^{-1}$ ), FT Esperança (4974 kg ha-1), P98Y51 (4954 kg ha $\mathrm{kg}^{-1}$ ), FTS 4188 (4941 kg ha-1) e M-SOY 9144RR (4850 kg ha-1), que não apresentaram diferenças significativas entre si. Em Gurupi (03/12), os maiores rendimentos foram obtidos por M-SOY 8527RR (3846 $\mathrm{kg} \mathrm{ha}^{-1}$ ), M-SOY 9988 (3665 $\left.\mathrm{kg} \mathrm{ha}^{-1}\right)$, M-SOY 9350 (3568 $\mathrm{kg} \mathrm{ha}^{-1}$ ) e P98R91 (3547 $\left.\mathrm{kg} \mathrm{ha}^{-1}\right)$.

Em Gurupi, as semeaduras atrasadas em relação à época normal, reduziram a produção de todas as cultivares, uma vez que a partir de março ocorreram maiores flutuações na temperatura e uma maior precipitação pluviométrica (FIG. 1) na fase de enchimento de grãos, propiciando o surgimento de doenças fúngicas de final de

Tabela 1 - Resumo da análise de variância conjuta de sete características agronômicas avaliadas em 21 cultivares de soja, na safra 2008/09, em Palmas e Gurupi, Tocantins

\begin{tabular}{|c|c|c|c|c|}
\hline \multirow{2}{*}{ FV } & \multirow{2}{*}{ GL } & \multicolumn{3}{|c|}{ Quadrados Médios } \\
\hline & & PG & TO & RO \\
\hline CULTIVAR & 20 & $1778767,5^{*}$ & $11,12^{*}$ & $92322,2 *$ \\
\hline EPOCA & 4 & $63886551,3^{*}$ & $201,84^{*}$ & $3507779,6^{*}$ \\
\hline CULTIVAR X ÉPOCA & 80 & $493687,28^{*}$ & $6,74^{*}$ & $26479,55^{*}$ \\
\hline BLOCO/ÉPOCA & 10 & 148049,34 & $7,94 *$ & $9501,96^{\mathrm{n} / \mathrm{s}}$ \\
\hline ERRO & 200 & 130349,88 & 3,42 & 8336,39 \\
\hline TOTAL & 314 & & & \\
\hline C.V (\%) & & 13,8 & 9,27 & 17,17 \\
\hline MÉDIA & & 2615,09 & 19,96 & 531,69 \\
\hline
\end{tabular}

PG: Produção de grãos $\left(\mathrm{kg} \mathrm{ha}^{-1}\right)$; TO: Teor de óleo (\%); RO: Redimento de óleo $\left(\mathrm{kg} \mathrm{ha}^{-1}\right)$; ${ }^{\text {ns: }}$ nao significativo; * significtivo ao nível de 5\% pelo teste $\mathrm{F}$ 
Tabela 2 - Médias de rendimento de grãos $\left(\mathrm{kg} \mathrm{ha}^{-1}\right)$ em 21 cultivares de soja, em cinco épocas de semeadura na safra 2008/2009, em Palmas e Gurupi, Tocantins

\begin{tabular}{|c|c|c|c|c|c|c|}
\hline & $\begin{array}{c}\text { GURUPI } \\
03 / 12 / 2008\end{array}$ & $\begin{array}{c}\text { GURUPI } \\
18 / 12 / 2008\end{array}$ & $\begin{array}{c}\text { GURUPI } \\
05 / 01 / 2009\end{array}$ & $\begin{array}{c}\text { PALMAS } \\
30 / 11 / 2008\end{array}$ & $\begin{array}{c}\text { PALMAS } \\
16 / 12 / 2008\end{array}$ & Médias \\
\hline P98Y70 & $2965 \mathrm{Bb}$ & $2259 \mathrm{Ca}$ & $1696 \mathrm{Db}$ & $3891 \mathrm{Ab}$ & $2475 \mathrm{Cb}$ & $2.657 \mathrm{~b}$ \\
\hline M-SOY 8766RR & $2911 \mathrm{Bb}$ & $1813 \mathrm{Ca}$ & $2074 \mathrm{Cb}$ & $4750 \mathrm{Aa}$ & $2741 \mathrm{Bb}$ & $2.858 \mathrm{a}$ \\
\hline M-SOY 9144RR & $2578 \mathrm{Cc}$ & $2374 \mathrm{Ca}$ & $1770 \mathrm{Db}$ & $4850 \mathrm{Aa}$ & $3333 \mathrm{Ba}$ & $2.981 \mathrm{a}$ \\
\hline BR/Emgopa 314 & $3388 \mathrm{Ba}$ & $1862 \mathrm{Da}$ & $1854 \mathrm{Db}$ & $4983 \mathrm{Aa}$ & $2600 \mathrm{Cb}$ & $2.937 \mathrm{a}$ \\
\hline P98R91 & $3547 \mathrm{Ba}$ & $1714 \mathrm{Ca}$ & $1666 \mathrm{Cb}$ & $5050 \mathrm{Aa}$ & $1941 \mathrm{Cc}$ & $2.784 \mathrm{~b}$ \\
\hline P98Y51 & $2937 \mathrm{Bb}$ & $1435 \mathrm{Da}$ & $1931 \mathrm{Db}$ & $4954 \mathrm{Aa}$ & $2350 \mathrm{Cb}$ & $2.721 \mathrm{~b}$ \\
\hline M-SOY 9988 & $3665 \mathrm{Aa}$ & $1955 \mathrm{Ba}$ & $1681 \mathrm{Bb}$ & $3712 \mathrm{Ab}$ & $2300 \mathrm{Bb}$ & $2.662 \mathrm{~b}$ \\
\hline P99R01 & $2612 \mathrm{Bc}$ & $1638 \mathrm{Ca}$ & $1982 \mathrm{Cb}$ & $4191 \mathrm{Ab}$ & $2333 \mathrm{Bb}$ & $2.551 \mathrm{c}$ \\
\hline M-SOY 8867RR & $3151 \mathrm{Bb}$ & $2350 \mathrm{Ca}$ & $1398 \mathrm{Db}$ & $3758 \mathrm{Ab}$ & $1808 \mathrm{Dc}$ & $2.493 \mathrm{c}$ \\
\hline M-SOY 9056RR & $3281 \mathrm{Bb}$ & $1684 \mathrm{Cb}$ & $1884 \mathrm{Cb}$ & $3995 \mathrm{Ab}$ & $1716 \mathrm{Cc}$ & $2.512 \mathrm{c}$ \\
\hline M-SOY 8527RR & $3846 \mathrm{Ba}$ & $1869 \mathrm{Ca}$ & $2197 \mathrm{Cb}$ & $4758 \mathrm{Aa}$ & $2466 \mathrm{Cb}$ & $3.027 \mathrm{a}$ \\
\hline M-SOY 8360RR & $3145 \mathrm{Bb}$ & $1533 \mathrm{Cb}$ & $2048 \mathrm{Cb}$ & $4008 \mathrm{Ab}$ & $1866 \mathrm{Cc}$ & $2.520 \mathrm{c}$ \\
\hline FT Esperança & $2134 \mathrm{Bd}$ & $1891 \mathrm{Ba}$ & $1586 \mathrm{Bb}$ & $4974 \mathrm{Aa}$ & $1987 \mathrm{Bc}$ & $2.515 \mathrm{c}$ \\
\hline FTS 4188 & $3100 \mathrm{Bb}$ & $2257 \mathrm{Ca}$ & $2668 \mathrm{Ca}$ & $4941 \mathrm{Aa}$ & $2541 \mathrm{Cb}$ & $3.101 \mathrm{a}$ \\
\hline CM 015 & $2424 \mathrm{Bc}$ & $1554 \mathrm{Cb}$ & $1382 \mathrm{Cb}$ & $3950 \mathrm{Ab}$ & $2158 \mathrm{Bc}$ & $2.293 \mathrm{~d}$ \\
\hline CM 017 & $2724 \mathrm{Bc}$ & $1134 \mathrm{Dc}$ & $1662 \mathrm{Cb}$ & 3316 Ac & $1954 \mathrm{Cc}$ & $2.158 \mathrm{~d}$ \\
\hline CM 136 & $1621 \mathrm{Bd}$ & $660 \mathrm{Dc}$ & $1296 \mathrm{Bb}$ & $3933 \mathrm{Ab}$ & $1783 \mathrm{Bc}$ & $1.859 \mathrm{e}$ \\
\hline CM 149 & $2461 \mathrm{Bc}$ & $1620 \mathrm{Cb}$ & $1755 \mathrm{Cb}$ & $3225 \mathrm{Ac}$ & $1958 \mathrm{Cc}$ & $2.204 \mathrm{~d}$ \\
\hline CM 102 & $2415 \mathrm{Ac}$ & $1368 \mathrm{Bb}$ & $2157 \mathrm{Ab}$ & $2850 \mathrm{Ac}$ & $1791 \mathrm{Bc}$ & $2.116 \mathrm{~d}$ \\
\hline NIDERA A 7002 & $3184 \mathrm{Bb}$ & $2240 \mathrm{Ca}$ & $2851 \mathrm{Ba}$ & $4520 \mathrm{Aa}$ & $2441 \mathrm{Cb}$ & $3.047 \mathrm{a}$ \\
\hline M-SOY 9350 & $3568 \mathrm{Aa}$ & $1555 \mathrm{Cb}$ & $2691 \mathrm{Ba}$ & $4058 \mathrm{Ab}$ & $2679 \mathrm{Bb}$ & $2.910 \mathrm{a}$ \\
\hline Médias & $2936 \mathrm{~B}$ & $1751 \mathrm{D}$ & $1916 \mathrm{~B}$ & $4222 \mathrm{~A}$ & $2249 \mathrm{C}$ & \\
\hline
\end{tabular}

Médias seguidas por uma mesma letra maiúscula na linha e minúscula na coluna, não diferem entre si, ao nível de 5\% pelo teste de Scott-Knott

ciclo. Além disso, nesta mesma fase, a maior precipitação resultou em menor radiação fotossintética para as plantas por efeito da baixa luminosidade. Barros et al. (2003) e Peluzio et al. (2005) também observaram reduções na produção de grãos com o retardamento da semeadura da soja, os quais atribuíram a redução no porte das plantas pelo encurtamento da fase vegetativa.

Os valores do rendimento (relativos e máximos) de grãos (TAB. 3) retratam a importância da época de semeadura para a cultura da soja, uma vez que podem resultar em redução de até $84 \%$ na produção de grãos. Convencionalmente, adotam-se, como critério de aceitação de produções, os valores até $80 \%$ da produção máxima de cada cultivar. Assim, todas as cultivares poderiam ser semeadas em Palmas em 30/11 e as cultivares P98Y70, P98R91, M-SOY 9988, M-SOY 8867RR, M-SOY 9056RR, M-SOY 8527RR, MSOY 8360RR, CM 017, CM 140, CM 102, NIDERA A7002 e M-SOY 9350 serem semeadas em Gurupi em 03/12.

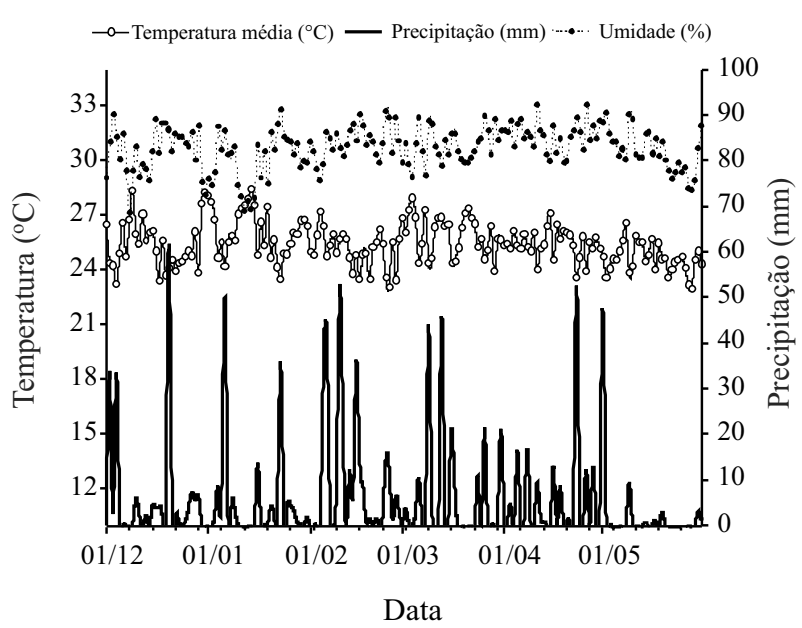

Figura 1 - Médias da umidade relativa do ar, temperatura e precipitação no período de dezembro de 2008 a maio de 2009 , em Gurupi-TO 
Tabela 3 - Rendimento de grãos (relativa e máxima) em 21 cultivares de soja, em cinco épocas de semeadura na safra 2008/2009, em Palmas e Gurupi, Tocantins

\begin{tabular}{ccccccc}
\hline & GURUPI & GURUPI & GURUPI & PALMAS & PALMAS & $\begin{array}{c}\text { Produção } \\
\text { máxima }\left(\mathrm{kg} \mathrm{ha}^{-1}\right)\end{array}$ \\
\hline P3/12/2008 & $18 / 12 / 2008$ & $05 / 01 / 2009$ & $30 / 11 / 2008$ & $16 / 12 / 2008$ & 3.891 \\
M-SOY 8766RR & 76,20 & 58,06 & 43,59 & 100,00 & 63,61 & 4.750 \\
M-SOY 9144RR & 53,15 & 48,28 & 43,09 & 100,00 & 57,71 & 4.850 \\
BR/Emgopa 314 & 67,00 & 37,37 & 37,21 & 100,00 & 52,18 & 4.983 \\
P98R91 & 70,24 & 33,00 & 32,00 & 100,00 & 38,44 & 5.050 \\
P98Y51 & 59,29 & 29,00 & 39,00 & 100,00 & 47,44 & 4.954 \\
M-SOY 9988 & 98,73 & 52,67 & 45,29 & 100,00 & 62,00 & 3.712 \\
P99R01 & 62,32 & 39,08 & 47,29 & 100,00 & 55,67 & 4.191 \\
M-SOY 8867RR & 83,85 & 62,53 & 37,20 & 100,00 & 48,11 & 3.758 \\
M-SOY 9056RR & 82,13 & 42,15 & 47,16 & 100,00 & 43,00 & 3.995 \\
M-SOY 8527RR & 80,83 & 39,28 & 46.17 & 100,00 & 51,83 & 4.758 \\
M-SOY 8360RR & 78,47 & 38,25 & 51,10 & 100,00 & 46,56 & 4.008 \\
FT Esperança & 42,90 & 38,02 & 31,89 & 100,00 & 40,00 & 4.974 \\
FTS 4188 & 62,74 & 45,67 & 54,00 & 100,00 & 51,42 & 4.941 \\
CM 015 & 61,36 & 39,34 & 35,00 & 100,00 & 54,63 & 3.950 \\
CM 017 & 82.14 & 34,19 & 50,12 & 100,00 & 59,00 & 3.316 \\
CM 136 & 41,21 & 16.78 & 33,00 & 100,00 & 45,33 & 3.933 \\
CM 149 & 76,31 & 50,23 & 54,41 & 100,00 & 60,71 & 3.225 \\
CM 102 & 84,73 & 48,00 & 75,68 & 100,00 & 62,84 & 2.850 \\
NIDERA A 7002 & 70,44 & 49,55 & 63,07 & 100,00 & 54,00 & 4.520 \\
M-SOY 9350 & 88,00 & 38,31 & 66,31 & 100,00 & 66,00 & 4.058 \\
\hline & & & & & & \\
\hline
\end{tabular}

\section{Teor de Óleo}

Para a característica teor de óleo (TAB. 4), os plantios realizados em Palmas (30/11 e 18/12) resultaram em maiores teores de óleo com, respectivamente, 21,47 e $21,62 \%$, sem, contudo, apresentarem diferenças significativas entre si. As cultivares que mais se destacaram, em ambas as épocas, foram: NIDERA A 7002, M-SOY 9144RR, BR/Emgopa 314, P98Y51 e FTS 4188. Para os plantios realizados em Gurupi, não foram detectadas diferenças significativas entre as cultivares na primeira época. As cultivares que mais se destacaram nestas épocas foram: M-SOY 8766RR, BR/Emgopa 314, P98R91 e CM 149.
De modo geral, observou-se uma tendência de redução no teor de óleo com o retardamento da semeadura, similarmente ao obtido com rendimento de grãos (TAB. 2). Tal redução, provavelmente, ocorreu devido principalmente às menores temperaturas e maiores precipitações que ocorreram aos 20-40 dias antes da maturação dos grãos, no plantio realizado em 05/01, em relação ao plantio realizado em 03/12 em Gurupi (FIG. 1). Em Palmas, não foram detectadas diferenças significativas para teor de óleo, para a grande maioria das cultivares, uma vez que as temperaturas médias e precipitação foram similares próximas à fase de maturação dos grãos (FIG. 2). 
Tabela 4 - Valores médios de teor de óleo (\%), em 21 cultivares de soja, em cinco épocas de semeadura, na safra 2008/2009, em Palmas e Gurupi, Tocantins

\begin{tabular}{|c|c|c|c|c|c|c|}
\hline & $\begin{array}{c}\text { GURUPI } \\
03 / 12 / 2008 \\
\end{array}$ & $\begin{array}{c}\text { GURUPI } \\
18 / 12 / 2008 \\
\end{array}$ & $\begin{array}{c}\text { GURUPI } \\
05 / 01 / 2009 \\
\end{array}$ & $\begin{array}{c}\text { PALMAS } \\
30 / 11 / 2008\end{array}$ & $\begin{array}{c}\text { PALMAS } \\
16 / 12 / 2008 \\
\end{array}$ & Médias \\
\hline P98Y70 & $20,13 \mathrm{Aa}$ & $18,19 \mathrm{Ab}$ & $16,73 \mathrm{Bb}$ & $19,13 \mathrm{Ab}$ & $20,75 \mathrm{Ab}$ & $19,99 \mathrm{~b}$ \\
\hline M-SOY 8766RR & $20,00 \mathrm{Aa}$ & $18,49 \mathrm{Ab}$ & $18,41 \mathrm{Aa}$ & $21,38 \mathrm{Aa}$ & $21,15 \mathrm{Ab}$ & $19,88 \mathrm{~b}$ \\
\hline M-SOY 9144RR & $21,73 \mathrm{Aa}$ & $19,72 \mathrm{Ba}$ & $17,22 \mathrm{Bb}$ & $22,87 \mathrm{Aa}$ & $23,75 \mathrm{Aa}$ & $21,06 \mathrm{a}$ \\
\hline BR/Emgopa 314 & $19,65 \mathrm{Ba}$ & $21,81 \mathrm{Aa}$ & $17,80 \mathrm{Ba}$ & $22,91 \mathrm{Aa}$ & $23,31 \mathrm{Aa}$ & $21,09 \mathrm{a}$ \\
\hline P98R91 & $19,71 \mathrm{Aa}$ & $19,53 \mathrm{Aa}$ & $17,14 \mathrm{Bb}$ & $21,70 \mathrm{Aa}$ & $17,97 \mathrm{Bb}$ & $19,21 \mathrm{~b}$ \\
\hline P98Y51 & $19,84 \mathrm{Ba}$ & $18,71 \mathrm{Bb}$ & $19,77 \mathrm{Ba}$ & $22,35 \mathrm{Aa}$ & $23,91 \mathrm{Aa}$ & $20,91 \mathrm{a}$ \\
\hline M-SOY 9988 & $21,09 \mathrm{Aa}$ & $20,04 \mathrm{Aa}$ & $16,17 \mathrm{Bb}$ & $22,74 \mathrm{Aa}$ & $19,25 \mathrm{Ab}$ & $19,85 \mathrm{~b}$ \\
\hline P99R01 & $22,06 \mathrm{Aa}$ & $20,49 \mathrm{Aa}$ & $16,22 \mathrm{Bb}$ & $20,77 \mathrm{Aa}$ & $19,99 \mathrm{Ab}$ & $19,90 \mathrm{~b}$ \\
\hline M-SOY 8867RR & $21,36 \mathrm{Aa}$ & $16,46 \mathrm{Bb}$ & $18,26 \mathrm{Ba}$ & $21,37 \mathrm{Aa}$ & $23,23 \mathrm{Aa}$ & $20,13 \mathrm{~b}$ \\
\hline M-SOY 9056RR & $21,74 \mathrm{Aa}$ & $16,99 \mathrm{Bb}$ & $15,75 \mathrm{Bb}$ & $23,55 \mathrm{Aa}$ & $21,95 \mathrm{Ab}$ & $20,00 \mathrm{~b}$ \\
\hline M-SOY 8527RR & $21,87 \mathrm{Aa}$ & $17,43 \mathrm{Bb}$ & $19,35 \mathrm{Ba}$ & $18,47 \mathrm{Bb}$ & $20,36 \mathrm{Ab}$ & $19,49 \mathrm{~b}$ \\
\hline M-SOY 8360RR & 20,19 Аa & $15,50 \mathrm{Bb}$ & $18,44 \mathrm{Aa}$ & $19,14 \mathrm{Ab}$ & $20,54 \mathrm{Ab}$ & $18,76 \mathrm{~b}$ \\
\hline FT Esperança & $19,94 \mathrm{Aa}$ & $16,72 \mathrm{Bb}$ & $16,59 \mathrm{Bb}$ & $19,12 \mathrm{Ab}$ & $19,94 \mathrm{Ab}$ & $18,46 \mathrm{~b}$ \\
\hline FTS 4188 & $19,30 \mathrm{Ba}$ & $18,04 \mathrm{Bb}$ & $21,16 \mathrm{Aa}$ & $22,54 \mathrm{Aa}$ & $22,89 \mathrm{Aa}$ & $20,78 \mathrm{a}$ \\
\hline CM 015 & $21,19 \mathrm{Aa}$ & $17,48 \mathrm{Bb}$ & $17,46 \mathrm{Bb}$ & $22,42 \mathrm{Aa}$ & $19,65 \mathrm{Ab}$ & $19,64 \mathrm{~b}$ \\
\hline CM 017 & $20,50 \mathrm{Aa}$ & $18,43 \mathrm{Bb}$ & $17,69 \mathrm{Bb}$ & $21.90 \mathrm{Aa}$ & $22,11 \mathrm{Aa}$ & $20,12 b$ \\
\hline CM 136 & $21,20 \mathrm{Aa}$ & $18,70 \mathrm{Bb}$ & $17,93 \mathrm{Ba}$ & $22,05 \mathrm{Aa}$ & $23,57 \mathrm{Aa}$ & $20,69 a$ \\
\hline CM 149 & $20,24 \mathrm{Aa}$ & $21,09 \mathrm{Aa}$ & $20,38 \mathrm{Aa}$ & $19,84 \mathrm{Ab}$ & $22,78 \mathrm{Aa}$ & $20,86 \mathrm{a}$ \\
\hline CM 102 & $19,85 \mathrm{Aa}$ & $17,99 \mathrm{Ab}$ & $14,09 \mathrm{Bb}$ & $19,92 \mathrm{Ab}$ & $20,49 \mathrm{Ab}$ & $18,47 \mathrm{~b}$ \\
\hline NIDERA A 7002 & $18,35 \mathrm{Ba}$ & $22,24 \mathrm{Aa}$ & $17,94 \mathrm{Ba}$ & $22,41 \mathrm{Aa}$ & $24,57 \mathrm{Aa}$ & $21,10 \mathrm{a}$ \\
\hline M-SOY 9350 & $21,36 \mathrm{Aa}$ & $15,77 \mathrm{Bb}$ & $16,33 \mathrm{Bb}$ & $23,34 \mathrm{Aa}$ & $21,68 \mathrm{Ab}$ & $19,70 \mathrm{~b}$ \\
\hline Médias & $20,56 \mathrm{~B}$ & $18,56 \mathrm{C}$ & $17,62 \mathrm{D}$ & $21,47 \mathrm{~A}$ & $21,62 \mathrm{~A}$ & \\
\hline
\end{tabular}

Médias seguidas por uma mesma letra maiúscula na linha e minúscula na coluna, não diferem entre si, ao nível de 5\% pelo teste de Scott-Knott

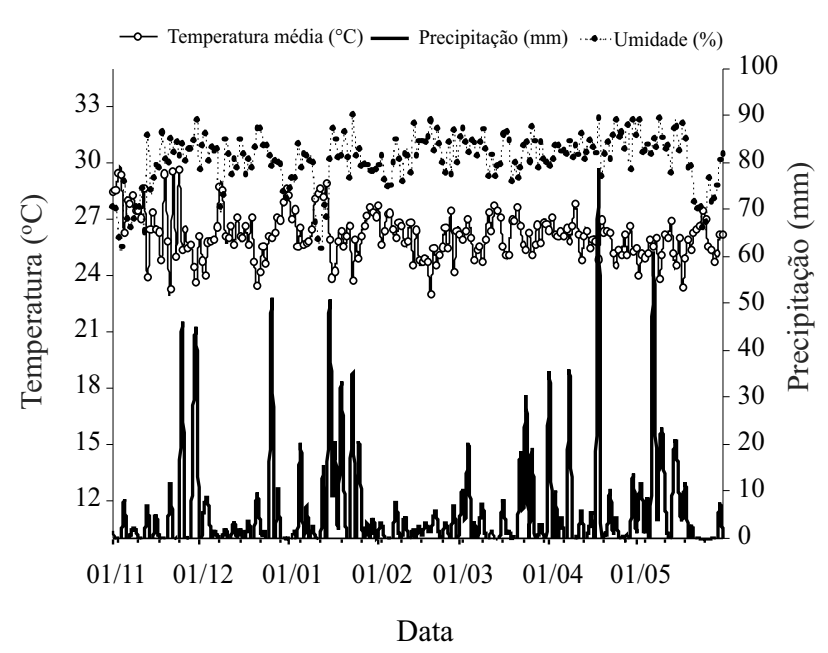

Figura 2 - Médias da umidade relativa do ar, temperatura e precipitação no período de dezembro de 2008 a maio de 2009 , em Palmas-TO
Estes resultados estão em concordância com aqueles obtidos por Albrecht et al. (2008), Horan (1974), Minuzzi et al. (2009), Pípolo (2002) e Rangel et al. (2004), que também observaram os efeitos das altas temperaturas e reduções hídricas no incremento do teor de óleo em soja. Entretanto, Maehler et al. (2003) ao trabalharem com duas cultivares de soja, em dois regimes hídricos (irrigado e não irrigado), verificaram que o teor de óleo nos grãos não foi alterado.

As temperaturas mais altas e as menores incidências de precipitação, na fase final de enchimento dos grãos, promoveram um comportamento diferencial entre as cultivares que, provavelmente, acarretaram em distúrbios bioquímicos nas biossínteses de óleo (ALBRECHT et al., 2008).

\section{Rendimento de Óleo}

Para a característica rendimento de óleo (TAB. 5), o plantio realizado em Palmas (30/11), independentemente da 
Tabela 5 - Rendimento de óleo $\left(\mathrm{kg} \mathrm{ha}^{-1}\right)$, em 21 cultivares de soja, em cinco épocas de semeadura, na Safra 2008/2009, em Palmas e Gurupi, Tocantins

\begin{tabular}{|c|c|c|c|c|c|c|}
\hline & $\begin{array}{c}\text { GURUPI } \\
03 / 12 / 2008 \\
\end{array}$ & $\begin{array}{c}\text { GURUPI } \\
18 / 12 / 2008\end{array}$ & $\begin{array}{c}\text { GURUPI } \\
05 / 01 / 2009\end{array}$ & $\begin{array}{c}\text { PALMAS } \\
30 / 11 / 2008\end{array}$ & $\begin{array}{c}\text { PALMAS } \\
16 / 12 / 2008\end{array}$ & Médias \\
\hline P98Y70 & $617,5 \mathrm{Ab}$ & $410,3 \mathrm{Ba}$ & $284,0 \mathrm{Cb}$ & $742,0 \mathrm{Ad}$ & $513,3 \mathrm{Bc}$ & $506,0 \mathrm{c}$ \\
\hline M-SOY 8766RR & $580,7 \mathrm{Bb}$ & $335,7 \mathrm{Ca}$ & $381,7 \mathrm{Cb}$ & $1.014,7 \mathrm{Ab}$ & $579,0 \mathrm{Bb}$ & $578,3 \mathrm{~b}$ \\
\hline M-SOY 9144RR & $608,0 \mathrm{Cb}$ & $489,0 \mathrm{Ca}$ & $304,7 \mathrm{Db}$ & $946,7 \mathrm{Ab}$ & $789,7 \mathrm{Ba}$ & $627,6 \mathrm{a}$ \\
\hline BR/Emgopa 314 & $655,7 \mathrm{Ba}$ & $409,7 \mathrm{Ca}$ & $328,3 \mathrm{Cb}$ & $1.142,3 \mathrm{Aa}$ & $655,7 \mathrm{Bb}$ & $628,5 \mathrm{a}$ \\
\hline P98R91 & $691,0 \mathrm{Ba}$ & $335,0 \mathrm{Ca}$ & $285,0 \mathrm{Cb}$ & $1.095,0 \mathrm{Aa}$ & $346,7 \mathrm{Cc}$ & $550,5 \mathrm{~b}$ \\
\hline P98Y51 & $581,3 \mathrm{Bb}$ & $269,3 \mathrm{Cb}$ & $362,3 \mathrm{Cb}$ & $1.105,3 \mathrm{Aa}$ & $565,3 \mathrm{Bb}$ & $576,7 \mathrm{~b}$ \\
\hline M-SOY 9988 & $770,7 \mathrm{Aa}$ & $392,3 \mathrm{Ba}$ & $272,0 \mathrm{Bb}$ & $844,0 \mathrm{Ac}$ & $441,3 \mathrm{Bc}$ & $544,1 \mathrm{~b}$ \\
\hline P99R01 & $595,3 \mathrm{Bb}$ & $334,0 \mathrm{Ca}$ & $334,0 \mathrm{C} \mathrm{b}$ & $872,3 \mathrm{Ac}$ & $466,3 \mathrm{Bc}$ & $520,4 \mathrm{c}$ \\
\hline M-SOY 8867RR & $674,3 \mathrm{Aa}$ & $385,7 \mathrm{Ba}$ & $257,7 \mathrm{Bb}$ & $803,7 \mathrm{Ac}$ & $420,3 \mathrm{Bc}$ & $508,3 \mathrm{c}$ \\
\hline M-SOY 9056RR & $711,3 \mathrm{Ba}$ & $286,3 \mathrm{Cb}$ & $298,7 \mathrm{Cb}$ & $942,0 \mathrm{Ab}$ & $376,3 \mathrm{Cc}$ & $522,9 \mathrm{c}$ \\
\hline M-SOY 8527RR & $835,0 \mathrm{Aa}$ & $273,3 \mathrm{Ba}$ & $425,3 \mathrm{Bb}$ & $925,7 \mathrm{Ab}$ & $500,7 \mathrm{Bc}$ & $602,8 \mathrm{~b}$ \\
\hline M-SOY 8360RR & $636,7 \mathrm{Aa}$ & $250,3 \mathrm{Bb}$ & $368,3 \mathrm{Ba}$ & $769,3 \mathrm{Ac}$ & $385,3 \mathrm{Bc}$ & $481,8 \mathrm{c}$ \\
\hline FT Esperança & $426,0 \mathrm{Bc}$ & $316,0 \mathrm{Ba}$ & $265,7 \mathrm{Bb}$ & $947,0 \mathrm{Ab}$ & $395,0 \mathrm{Bc}$ & $469,9 \mathrm{c}$ \\
\hline FTS 4188 & $600,7 \mathrm{Bb}$ & $408,3 \mathrm{Ca}$ & $557,0 \mathrm{Bb}$ & $1.123,7 \mathrm{Aa}$ & $583,0 \mathrm{Bb}$ & $654,5 \mathrm{a}$ \\
\hline CM 015 & $514,0 \mathrm{Bc}$ & $271,0 \mathrm{Cb}$ & $242,3 \mathrm{Ca}$ & $887,0 \mathrm{Ac}$ & $420,7 \mathrm{Bc}$ & $467,0 \mathrm{c}$ \\
\hline CM 017 & $560,0 \mathrm{Bb}$ & $207,7 \mathrm{Db}$ & $293,7 \mathrm{Db}$ & $716,3 \mathrm{Ad}$ & $426,7 \mathrm{Cc}$ & $440,9 \mathrm{~d}$ \\
\hline CM 136 & $346,3 \mathrm{Bc}$ & $123,7 \mathrm{Cb}$ & $224,3 \mathrm{Cb}$ & $868,0 \mathrm{Ac}$ & $420,0 \mathrm{Bc}$ & $396,5 \mathrm{~d}$ \\
\hline CM 149 & $498,3 \mathrm{Bc}$ & $341,0 \mathrm{Ba}$ & $361,0 \mathrm{Bb}$ & $642,7 \mathrm{Ad}$ & $445,3 \mathrm{Bc}$ & $457,7 \mathrm{c}$ \\
\hline CM 102 & $480,3 \mathrm{Ac}$ & $243,7 \mathrm{Bb}$ & $307,0 \mathrm{Bb}$ & $562,0 \mathrm{Ad}$ & $366,0 \mathrm{Bc}$ & $391,8 \mathrm{~d}$ \\
\hline NIDERA A 7002 & $584,3 \mathrm{Bb}$ & $492,0 \mathrm{Ba}$ & $513,0 \mathrm{Ba}$ & $1.020,0 \mathrm{Ab}$ & $601,0 \mathrm{Bb}$ & $642,1 \mathrm{a}$ \\
\hline M-SOY 9350 & $765,0 \mathrm{Ba}$ & $245,3 \mathrm{Db}$ & $439,7 \mathrm{Ca}$ & $944,3 \mathrm{Ab}$ & $582,3 \mathrm{Cb}$ & $595,4 \mathrm{~b}$ \\
\hline Médias & $605,9 \mathrm{~B}$ & $327,3 \mathrm{C}$ & $338,4 \mathrm{C}$ & $900,7 \mathrm{~A}$ & $487,1 \mathrm{C}$ & \\
\hline
\end{tabular}

Médias seguidas por uma mesma letra maiúscula na linha e minúscula na coluna, não diferem entre si, ao nível de 5\% pelo teste de Scott-Knott

cultivar, alcançou a maior média geral $\left(900,7 \mathrm{~kg} \mathrm{ha}^{-1}\right)$, seguido do plantio efetuado em Gurupi (03/12) $\left(605,9 \mathrm{~kg} \mathrm{ha}^{-1}\right)$. Já no plantio realizado em 30/11, a cultivar BR/EMGOPA se destacou com a maior média de rendimento de óleo (1142,3 $\left.\mathrm{kg} \mathrm{ha}^{-1}\right)$, seguida de FTS 4188 (1123,7 $\left.\mathrm{kg} \mathrm{ha}^{-1}\right)$, P98R91 (1095 kg ha-1), NIDERA A $7002\left(1020,0 \mathrm{~kg} \mathrm{ha}^{-1}\right)$ e M-SOY 8766RR (1014,7 $\left.\mathrm{kg} \mathrm{ha}^{-1}\right)$, que não apresentaram diferenças significativas entre si. Em Gurupi (03/12), as maiores produções foram obtidas por M-SOY $8527 \mathrm{RR}$ (835 kg ha-1), M-SOY 9988 (770,7 kg ha-1) e M-SOY $9350\left(765 \mathrm{~kg} \mathrm{ha}^{-1}\right)$. Não foram detectadas diferenças significativas, entre estas épocas, para MSOY 9988, MSOY 8867RR e CM 102. Os menores rendimentos de óleo foram obtidos nos plantios realizados em Gurupi, nos períodos de 18/12 e 05/01.

Com o conjunto de dados contidos nas Tabelas 2; 3 e 5 , pode-se observar que a época de semeadura mais favorável à produção de grãos também favorece ao rendimento de óleo. Segundo Montaño-Velasco (1994), cultivares com maior produtividade de grãos também apresentam maior rendimento de óleo, uma vez que ocorre associação positiva e significativa entre os mesmos $(R=0,99)$. Assim, em programas de melhoramento, a obtenção de alta produtividade de óleo pode ser obtida através de uma seleção indireta para produtividade de grãos.

\section{Conclusões}

1. Foram detectadas diferenças significativas entre cultivares, épocas e cultivares $\mathrm{x}$ épocas para todas as características;

2. Para as características rendimento de grãos, teor de óleo e rendimento de óleo, de modo geral, a primeira época de plantio em Palmas (30/11) e em Gurupi (03/12), são as mais propícias; 
3. Temperaturas mais altas e menores médias de precipitação, durante a fase de enchimento de grãos, favorecem ao acúmulo de teor de óleo nos grãos;

4. As cultivares comerciais NIDERA A 7002, MSOY 9144RR, BR/EMGOPA 314, P98Y51 e FTS 4188 são as que mais se destacam para a produção de biocombustível.

\section{Agradecimentos}

Ao Conselho Nacional de Desenvolvimento Científico e Tecnológico (CNPq), pelo apoio financeiro.

A Coordenação de Aperfeiçoamento de Pessoal de Nivel Superior (CAPES), pela concessão da bolsa no Programa de Mestrado em Agroenergia/UFT.

\section{Referências}

ALBRECHT, L. P. et al. Teores de óleo, proteínas e produtividade de soja em função da antecipação da semeadura na região oeste do Paraná. Bragantia, v. 67, n. 04, p. 865-873, 2008.

BARROS, H. B et al. Efeito das épocas de semeadura no comportamento de cultivares de soja no Sul do Estado do Tocantins. Revista Ceres, v. 03, n. 02, p. 37-38, 2003.

CLEMENTE, T. E.; CAHOON, E. B. Soybean oil: genetic approaches for modification of functionality and total content. Plant physiology, v. 151, n. 03, p. 1030-1040, 2009.

CRUZ, C. D. ; REGAZZI, A. J. Modelos biométricos aplicados ao melhoramento genético. Viçosa, UFV: Imprensa Universitária, 2006. 390 p.

FEHR, W. R. et al. Stage of development descriptions for soybeans, Glycine max L. Merrill. Crop Science, v. 11, n. 06, p. 929-931, 1971.

FERRARI, R. A.; OLIVEIRA, V. S.; SACABIO, A. Biodiesel da soja - Taxa de conversão em ésteres etílicos, caracterização físico-química e consumo em gerador de energia. Química Nova, v. 28, n. 01, p. 19-23, 2005.

HORAN, F. E. Soy prontein products and their production. Journal of the American Oil Chemists, Society, n. 01, v. 51, p. $67-73,1974$

INSTITUTO ADOLFO LUTZ. Normas analíticas do Instituto Adolfo Lutz. São Paulo: Instituto Adolfo Lutz, 1985. p. 42-43. v. 1.
KINNEY, A. J.; CLEMENTE, T. E. Modifying soybean oil for enhanced performance in biodiesel blends. Fuel processing technology, v. 86, n. 02, p. 1137-1147, 2005.

MAEHLER, A. R. et al Qualidade de grãos de duas cultivares de soja em função da disponibilidade de água no solo e arranjo das plantas. Ciência Rural, v. 33, n. 02, p. 213-218, 2003.

MINUZZI, A. et al. Rendimento de teores de óleo e proteínas de quatro cultivares de soja, produzidas em dois locais no estado do Mato Grosso do Sul. Ciência e Agrotecnologia, v. 33, n. 04, p. 80-93, 2009.

MONTAÑO-VELASCO, J. C. Análise genética de populações $F 3$ de soja derivadas de cruzamentos em cadeia com ênfase na produção de óleo. 1994. 115 f. Tese (Doutorado em Genética e Melhoramento de Plantas) - Escola Superior de Agricultura "Luiz de Queiroz", Universidade de São Paulo, Piracicaba.

PELUZIO, J. M. et al. Comportamento de cultivares de soja no sul do estado do Tocantins. Bioscience Journal, v. 21, n. 03, p. 113-118, 2005.

PELUZIO, J. M. et al. Desempenho de cultivares de soja, em duas épocas de semeadura, no sul do Estado de Tocantins. Bioscience Journal, v. 22, n. 02, p. 69-74, 2006

PEREIRA, G. D. et al. Adaptabilidade e estabilidade de genótipos de soja avaliados para resistência ao oídio. Ciência Rural, v. 38, n. 07, p. 1836-1842, 2008.

PÍPOLO, A. E. Influência da temperatura sobre as concentrações de proteína e óleo em sementes de soja (Glycine max (L.) Merril). 2002. $128 \mathrm{f}$. Tese (Doutorado em Fitotecnia) - Escola Superior de Agricultura "Luiz de Queiroz", Universidade de São Paulo, Piracicaba.

RANGEL, M. A. S. et al. Efeito do genótipo e do ambiente sobre os teores de óleo e proteína nos grãos de soja, em quatro ambientes da Região Sul de Mato Grosso do Sul, safra 2002/2003. Dourados: Embrapa Agropecuária Oeste, 2004. 30 p. (Boletim de pesquisa e desenvolvimento, 17)

RAO, A. C. S. et al. Cultivar and climatic effects on the protein content of soft white winter wheat. Agronomy Journal, v. 85, n. 02, p. 1023-1028, 1993.

SILVA, P. R. F.; FREITAS, T. F. S. Biodisel: o ônus e o bônus de produzir combustível. Ciência Rural, v. 38, n. 03, p. 843-851, 2008.

SILVA, W. C. J.; DUARTE, J. B. Métodos estatísticos para estudo de adaptabilidade e estabilidade fenotípica em soja. Pesquisa Agropecuária Brasileira, v. 41, n. 01, p. 23-30, 2006. 